\title{
LIST OF PARTICIPANTS
}

D.C. ABBOTT, University of Wisconsin, Madison, USA

Y. ANDRILLAT, Observatoire de Haute Provence, St.Michel, France

M.J. BARLow, Anglo-Australian Observatory, Epping, New South Wales, Australia

W.P. BIDELMAN, Warner and Swasey Observatory, East Cleveland, USA

G.F. BISIACCHI, Instituto di Astronomia, Universidad de Mexico, Mexico

B. BOHANNAN, University of Colorado, Boulder, USA

C.T. BOLTON, David Dunlap Observatory, Richmond Hill, Canada

J. BREYSACHER, European Southern Observatory, Santiago, Chile

R. CARLBERG, University of British Columbia, Vancouver, Canada

L. CARRASCo, Instituto di Astronomia, Universidad de Mexico, MeXico

J.P. CASSINELLI, University of Wisconsin, Madison, USA

J.I. CASTOR, Joint Institute for Laboratory Astrophysics, Boulder, USA

C. CHIOSI, Instituto di Astronomia, Padova, Italy

P.S. CONTI, Joint Institute for Laboratory Astrophysics, Boulder, USA

R. COSTERo, Instituto di Astronomia, Universidad de Mexico, MeXico

A.P. COWLEY, University of Michigan, Ann Arbor, USA

D. DEARBORN, Steward Observatory/University of Arizona, TUCSOT, USA

E.L. VAN DESSEL, Royal Belgian Observatory, Brussels, Belgium

C.G. DAVIS, Las Alamos Scientific Laboratory, USA

A. DELGADO, Max Planck Institut für Physik und Astrophysik, Münich, FRG

D. EBBETS, University of Colorado, Boulder, USA

H.J. FALK, University of Western Ontario, London, Canada

C. FIRMANI, Instituto di Astronomia, Universidad de Mexico, MEXICO

C.D. GARMANY, University of Colorado, Boulder, USA

G. HAMMERSCHLAG-HENSBERGE, Astronomical Institute, Amsterdam, Netherlands

S. HEAP, Goddard Space Flight Center, Greenbelt, USA

A.G. HEARN, Sterrewacht Somnenborgh, Utrecht, Netherlands

H.F. HEINRICH, Astronomical Institute, Amsterdam, Netherlands

T.J. HERCZEG, University of Oklahoma, Norman, USA

E.P.J. VAN DEN HEUVEL, Astronomical Institute, Amsterdam, Netherlands

V.A. HUGHES, Queen's University, Kingston, Canada

D.G. HUMMER, Joint Institute for Laboratory Astrophysics, Boulder, USA

J.B. HUTCHINGS, Dominion Astrophysical Observatory, Victoria, Canada

A.R. HYLAND, Mt. Stromlo and Siding Spring observatory, Australia 
S. JEFFERS, York University, Downsview, Canada

M. KLUTZ, Institut d'Astrophysique, Liège, Belgium

P.B. KUNASZ, University of Colorado, Boulder, USA

S. KWOK, York University, Downsview, Canada

S.A. LAMB, University of California, Los Angeles, USA

H.J.G.L.M. LAMERS, Space Research Laboratory, Utrecht, Netherlands

P. LEDOUX, Institut d'Astrophysique, Liège, Belgium

E.M. LEEP, University of Washington, Seattle, USA

J.B. LESTER, University of Toronto, Mississauga, Canada

K.C. LEUNG, U.S. Department of Energy, Washington D.C., USA

C. DE LOORE, Astrofysisch Instituut, Brussels, Belgium

L.S. LUUD, Tartv Observatory, Estonia, USSR

B.T. LYNDS, Kitt Peak National Observatory, Tucson, USA

J.M. MARLBOROUGH, University of Western Ontario, London, Canada

P. MASSEY, University of Colorado, Boulder, USA

T.J. MAZUREK, University of Texas, Austin, USA

A.F.J. MOFFAT, Université de Montreal, Canada

N. MORRISON, Joint Institute for Laboratory Astrophysics, Boulder, USA

D.C. MORTON, Anglo-Australian Observatory, Epping, Australia

E. NASI, Instituto di Astronomia, Padova, Italy

V.S. NIEMELA, Instituto de Astronomia y Fisica del Espacio, Buenos Aires, Argentina

P. NOERDLINGER, University of Colorado, Boulder, USA

G. OLSON, Astrofysisch Instituut, Brussels, Belgium

M. OVENDEN, University of British Columbia, Vancouver, Canada

W. PACKET, Astrofysisch Instituut, Brussels, Belgium

S. PARSON, University of Texas, Austin, USA

P. PISMIS, Instituto di Astronomia, Universidad de Mexico, MeXico

M. PLAVEC, University of California, Los Angeles, USA

R. POECKERT, Dominion Astrophysical Observatory, Victoria, Canada

J. RAHE, Remeis Observatory, Bamberg, FRG

B. ROCCA-VOLMERANGE, Institut d'Astrophysique, Paris, France

D. SCHNEIDER, California Institute of Technology, Pasadena, USA

R. SCHOLES, Dominion Astrophysical Observatory, Victoria, Canada

W. SEGGEWISS, Observatorium Hoher List, Daun, FRG

P.R. SCHWARTZ, Naval Research Laboratory, Washington D.C., USA

T.P. SNOW, Jr., University of Colorado, Boulder, USA

R. SREENIVASAN, University of Calgary, Canada

R. STALIO, Osservatorio Astronomico di Trieste, Italy

J.P. SWINGS, Institut d'Astrophysique, Liège, Belgium

R.N. THOMAS, Institut d'Astrophysique, Paris, France

A.V. TUTUKoV, Astronomical Council, Academy of Sciences, MOSCOW, USSR 
A.B. UNDERHILL, Institut d'Astrophysique, Paris, France

D. VAN BLERKOM, Joint Institute for Laboratory Astrophysics, BOUlder, USA

D. VANBEVEREN, Astrofysisch Instituut, Brussels, Belgium

J.M. VREUX, Institut d'Astrophysique, Liège, Belgium

A.J. WILLIS, University College London, England

R.E. WILSON, University of South Florida, Tampa, USA

W.J.F. WILSON, University of Calgary, Calgary, Canada

B. WOLF, Landessternwarte Königstuhl, Heidelberg, FRG

S. WOLFF, Institute for Astronomy, University of Hawaii, USA

M. ZEILIK, University of New Mexico, Albuquerque, USA

J. ZIOLKOWSKI, Nicholas Copernicus Astronomical Center, Warsaw, Poland

CAMEO APPEARANCES BY:

C. AIKMAN, Dominion Astrophysical Observatory, Victoria, Canada

J. AUMAN, University of British Columbia, Vancouver, Canada

A. BATTEN, Dominion Astrophysical Observatory, Victoria, Canada

D. CRAMPTON, Dominion Astrophysical Observatory, Victoria, Canada

A. GOWER, University of Victoria, Canada

F.D.A. HARTWICK, University of Victoria, Canada

J. WOODROW, University of British Columbia, Vancouver, Canada 


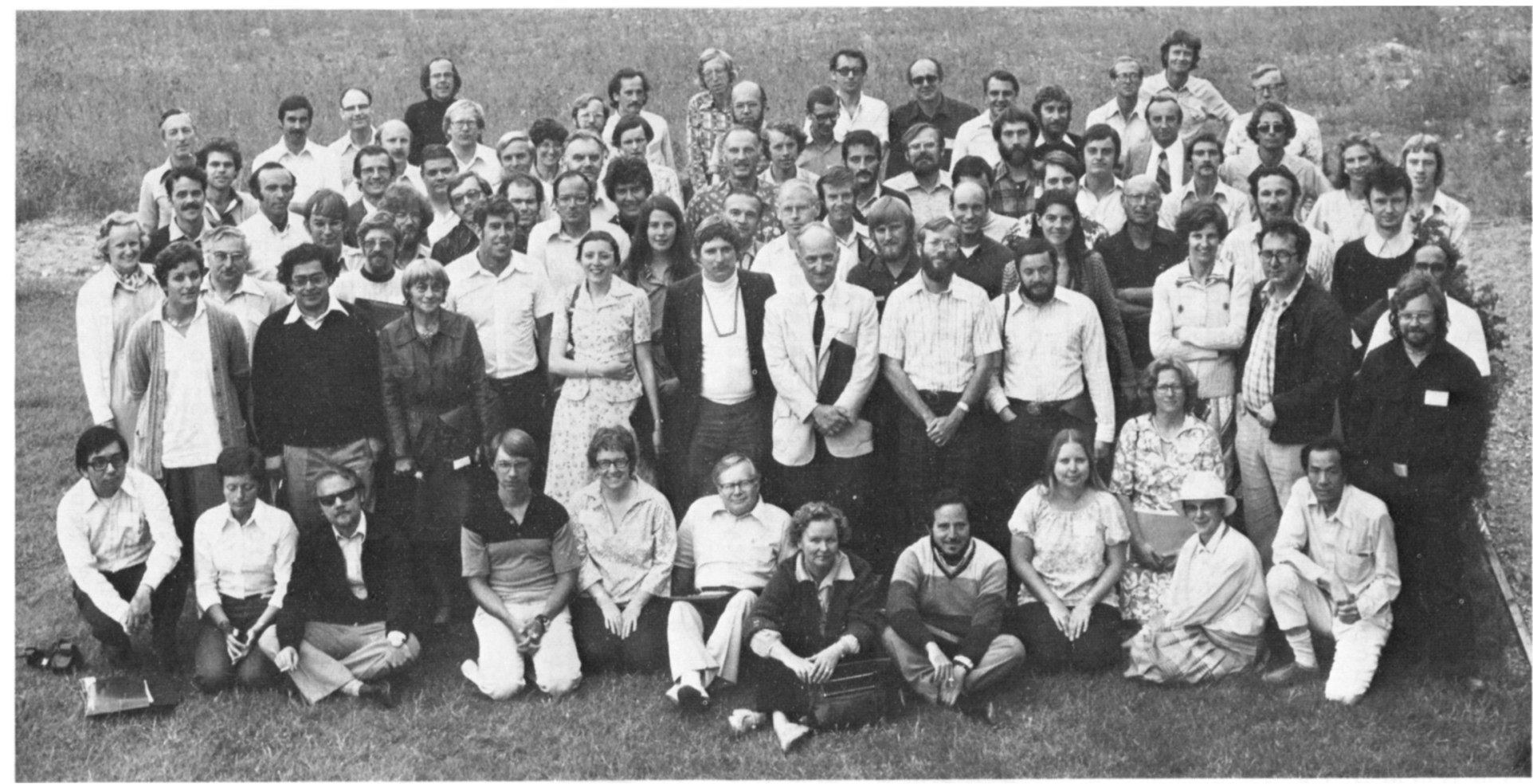




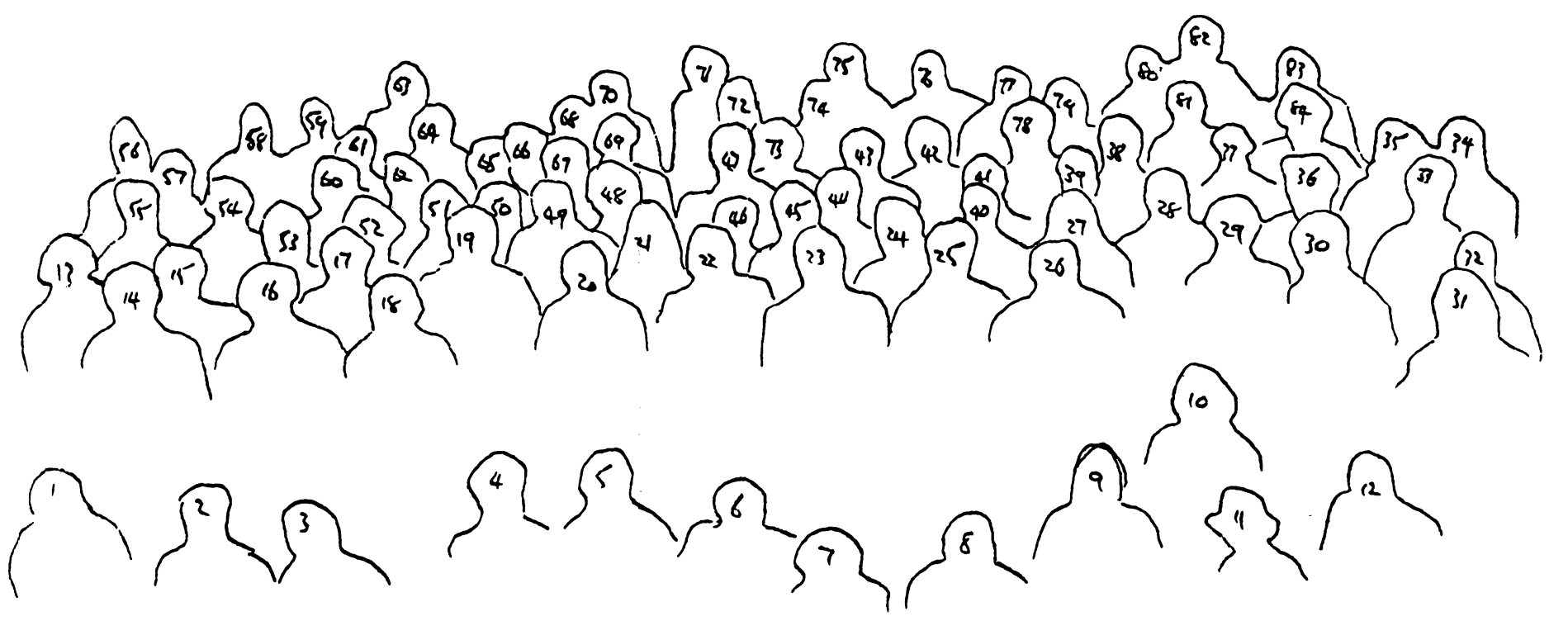

1. Kwok 2. Klutz 3. Bisiacchi 4. Ebbets 5. Wolff 6. Bidelman 7. Niemela 8. Costero 9. Leep 10. Heap 11. Underhill 12. Leung 13. Cowley 14. Rocca-Volmerange 15. Herczeg 16. Carrasco 17. Falk 18. Pismis 19. Snow 20. Hensberge 21. Lamb 22. Hyland 23. Ledoux 24. Schneider 25. Castor 26. Vreux 27. Morrison 28. De Loore 29. Nasi 30. Chiosi 31. Zeilik 32. Sreenivasan 33. Seggewiss 34. Scholes 35. Lynds 36. Conti 37. Willis 38. Vanbeveren 39. Bolton 40. Lester 41. Breysacher 42. Mazurek 43. Delgado 44. Wilson 45. Wilson 46. Andrillat 47. Noerdlinger 48. Andrillat 49. Van Blerkom 50. Massey 51. Poeckert 52. Abbott 53. Schwartz 54. Marlborough 55. Moffat 56. Hughes 57. Carlberg 58. Swings 59. Morton 60. Hearn 61. Parson 62. Luud 63. Jeffers 64. Cassinelli 65. Rahe 66. Garmany 67. Ovenden 68. Olson 69. Barlow 70. Tutukov 71. Van den Heuvel 72. Hummer 73. Packet 74. Ziolkowski 75. Stalio 76. Firmani 77. Wolf 78. Henrichs 79. Van Dessel 80. Kunasz 81. Plavec 82. Hutchings 83. Davis 84. Bohannan 